\title{
Quantum Zeno effect in atomic Bragg scattering
}

\author{
S. Dyrting ${ }^{1,2}$ and M. J. Gagen ${ }^{2}$ \\ ${ }^{1}$ Department of Physics, Hong Kong University of Science and Technology, \\ Clear Water Bay, Kowloon, Hong Kong, People's Republic of China \\ ${ }^{2}$ Department of Physics, The University of Queensland, St. Lucia, Queensland 4072, Australia
}

(Received 23 October 1996; revised manuscript received 1 April 1997)

\begin{abstract}
We propose an experiment to observe the quantum Zeno effect in atomic Bragg scattering. The quantum Zeno effect is the inhibition of the free evolution of a system when that system is subject to a decohering measurement process. This effect is usually studied theoretically in an idealized two-level system, though this system has been difficult to realize in practice. The coherent population exchange between the forward and Bragg beams provides an ideal two-level system, though as there is no practical scheme for continuously monitoring the motion of an atom we include decoherence by adding noise to the laser. Using the method of stochastic Hamiltonians we show that the noise suppresses tunneling in the two-state system and, in the large noise limit, the system can freeze in its initial state. [S1050-2947(97)00808-1]
\end{abstract}

PACS number(s): 42.50.Vk, 32.80.Pj

\section{INTRODUCTION}

Atom optics is an ideal testing ground for fundamental processes in quantum mechanics. Bose-Einstein condensation in a weakly interacting gas [1], the realization of the quantum kicked rotor and the subsequent observation of dynamic localization [2], and the recent observation of LandauZener tunneling in a linearly biased potential [3] are all examples of idealized quantum systems that have been realized in atom optics and corresponding fundamental processes observed. The preparation of quantum states through atomic cooling and trapping and the excellent control of the spatial and temporal variation of the optical and magnetic potentials have been central to this success.

In this article we are motivated to propose an experimental realization in atom optics of the quantum Zeno effect. The quantum Zeno effect is the inhibition of the free evolution of a system when that system is subject to a decohering measurement process [4-6]. Recently there has been one claimed experimental test of the Zeno effect by Itano et al. [7] performed on a hyperfine transition of laser cooled Be ions. This experiment realized a measurement of the two-level system by incorporating an additional optical cycling transition. Experiments on optical transitions have also been proposed $[8,9]$. However, in this case there is some difficulty in arranging for the measurement time scale to be much shorter than the time scales of any other nonunitary effects such as dissipation. This is necessary to cleanly display the quantum Zeno effect. In contrast, atom optics is in the opposite situation. Provided the light is sufficiently far from resonance, the atomic motion in a laser beam is very nearly conservative with only a very small irreversible component due to residual spontaneous emission.

The measurement process is modeled explicitly as a nonunitary interaction which can be either continuous or discontinuous, selective or nonselective, and either perfectly accurate or somewhat inaccurate. Itano et al. used a simple "collapse hypothesis"' to model an accurate sequence of discontinuous nonselective measurements. Later analysis [1012] demonstrated that the decohering processes must be con- sidered in any full treatment of the interaction between a system and the environment which includes the measurement apparatus. These results are in accord with the decoherence approach developed by Zurek [13] which show systems decohering in the pointer basis defined by the systemenvironment interaction.

It is not presently possible to continuously measure atomic center-of-mass observables, so we choose instead to add a classical noise source to mimic the decohering effects of a continuous, nonselective measurement process. In this paper we add random fluctuations to the spatial phase of a laser standing wave and average over the ensemble of noise trajectories. We emphasize that the decoherence process must be carefully matched to the coherently evolving system in order to give the Zeno effect. The choice of phase noise leads to decoherence in the momentum basis [14] thereby restricting consideration to systems featuring coherent tunneling between momentum eigenstates. Bragg scattering of atoms from a standing wave laser is such a system. Furthermore, first-order Bragg scattering realizes the ideal two-level model (TLM) with population coherently tunneling between the forward and Bragg beams [15]. The TLM is completely solvable, and its relation to the quantum Zeno effect has been studied in depth [6]. This paper continues the trend towards using atom optics to realize idealized quantum systems.

\section{SCATTERING WITH PHASE NOISE}

Consider the quantized motion of an atom in a laser standing wave with wave number $k_{L}$, frequency $\omega_{L}$, and phase $\phi$ measured relative to the fixed laboratory frame. We assume that the laser is nearly resonant with an internal atomic transition of frequency $\omega_{a}$. When the atom is excited by the field its internal state changes in energy by $\hbar \omega_{a}$ and its center-of-mass momentum along the laser axis changes by $\pm \hbar k_{L}$. When the detuning of the laser from the atomic transition $\delta_{L}=\omega_{L}-\omega_{a}$ is large compared to the transition lifetime and Rabi frequency $\Omega$ only virtual transitions into the excited state can occur. In this case the internal excited states can be adiabatically eliminated [16] and the center-of-mass 
motion is conservative and motion along the laser axis can be described by the Hamiltonian

$$
\hat{H}_{\phi}=\hat{p}^{2} / 2+\kappa \cos (\hat{q}-\phi) .
$$

Here we have transformed to dimensionless quantities. The operators $\hat{q}$ and $\hat{p}$ are the position and momentum of the atom along the laser axis and are scaled by $1 / 2 k$ and $2 \hbar k$, respectively. Time $t$ is measured in units of $1 / 8 \omega_{r}$ where $\omega_{r}=\hbar k_{L}^{2} / 2 m$ is the recoil frequency of the atom. The coupling $\kappa=\Omega^{2} / 64 \delta_{L} \omega_{r}$ is the rescaled effective Rabi frequency.

We are interested in the case where the phase of the standing wave changes in time. Experiments with timedependent phases have already been performed in the context of quantum chaos [17], Landau-Zener tunneling [3], and Bragg scattering [18]. In all these cases the phase was a simple function of time. In contrast to this, we consider the case where the phase undergoes a random walk from its initial value $\phi(0)=0$ and is given by $\phi=\sqrt{D} W$ where $D$ is the phase diffusion rate and $W=W(t)$ is the canonical Wiener process [19]. All information on the reproducible dynamics is then contained in the density operator $\hat{\rho}$ obtained by averaging over the ensemble of phase trajectories. In any experiment this is achieved by summing the results of a large number of runs, each subject to a different random trajectory for the phase.

From Eq. (2.1) it can be seen that the noise enters the dynamics in a highly nonlinear fashion. In general this prevents a closed solution for the position and momentum statistics. However, a considerable simplification results when only the momentum statistics are of interest. By making a time-dependent unitary transformation of the atomic state, the transformed Hamiltonian is linear in the noise. This transformation preserves momentum statistics but scrambles the position information. Using the method of stochastic Hamiltonians [14] the ensemble evolution is described by the master equation

$$
\frac{d \hat{\rho}}{d t}=-i\left[\hat{H}_{0}, \hat{\rho}\right]-\frac{D}{2}[\hat{p},[\hat{p}, \hat{\rho}]] .
$$

The first term in the above equation describes the systematic evolution of the atom in terms of the Hamiltonian of Eq. (2.1) with $\phi=0$. The effect of the double commutator is best illustrated by considering the evolution of $\hat{\rho}$ in the momentum representation $\rho\left(p, p^{\prime}\right)=\left\langle p|\hat{\rho}| p^{\prime}\right\rangle$. Ignoring the systematic evolution we find

$$
\frac{d \rho}{d t}\left(p, p^{\prime}\right)=-\frac{D}{2}\left(p-p^{\prime}\right)^{2} \rho\left(p, p^{\prime}\right) .
$$

Hence the double commutator has no effect on the momentum population $\rho(p, p)$ but causes momentum coherences $\rho\left(p, p^{\prime}\right), p \neq p^{\prime}$ to decay exponentially in time. For any individual atomic state trajectory the evolution is entirely coherent, but coherence is destroyed between trajectories. This leads the ensemble to decohere in the momentum basis.

As noted in Ref. [14] the master equation (2.2) also describes continuous nonselective measurement of atomic momentum. We emphasize that this equivalence is only with respect to the momentum statistics. This is of interest as it is presently impractical to perform direct measurements of atomic variables. The dual interpretation of the master equation (2.2) allows the phase noise ensemble to duplicate the evolution of an atomic system subject to momentum measurements. This novel equivalence allows us to model the quantum Zeno effect in atomic systems. The phase noise ensemble decoheres in the momentum basis and can thus model a Zeno effect whenever the system involves a coherent coupling of momentum states. This situation is precisely what occurs in first-order Bragg scattering.

\section{FIRST-ORDER SCATTERING}

First-order Bragg scattering occurs when a beam of atoms with momentum $-\hbar k_{L}$, typically called the forward beam, interacts with a plane standing wave and is strongly coupled to the state with momentum $+\hbar k_{L}$. This state is referred to as the Bragg beam [18]. As well as the plane wave condition, in order to ensure that the population in all other scattering orders remains small the intensity should satisfy $\kappa \ll 1 / 8$. This condition also indicates that Bragg scattering is a highly nonclassical effect. The boundary in momentum between classical oscillation and libration is defined by the maximum momentum on the separatrix $p_{\mathrm{sx}}=2 \kappa$ [20]. In dimensionless variables the forward and Bragg beams satisfy $p_{f}=-1 / 2<-p_{\mathrm{sx}}$ and $p_{b}=1 / 2>p_{\mathrm{sx}}$ which ensures there is no classical force coupling these states. In this paper we set $\kappa=0.1$. This corresponds to an effective Rabi frequency $\Omega_{\mathrm{eff}}=8 \omega_{r} / 10$.

In first-order Bragg scattering the momentum state $|p\rangle$ with $p \approx p_{f}$ (forward beam) is strongly coupled to the state $|p+1\rangle$ with $p+1 \approx p_{b}$ (Bragg beam). The kinetic energies of these two states are nearly degenerate, and hence they are strongly coupled. This leads to oscillations in the populations of the states $|p\rangle$ and $|p+1\rangle$ reflecting coherent tunneling across the separatrix. These oscillations are referred to as Pendellösung oscillations and have been observed in the Bragg beam populations [21].

This picture is somewhat complicated by the finite width in momentum of the initial ensemble of atoms. The initial atomic state is prepared in the mixture

$$
\hat{\rho}_{0}=\int_{-\infty}^{\infty} d p \wp(p)|p\rangle\langle p| .
$$

Here $\wp(p)$ is the distribution function for momentum in the incoming beam given by

$$
\wp(p)=\frac{1}{\sigma \sqrt{2 \pi}} e^{-\left(p-p_{f}\right)^{2} / 2 \sigma^{2}} .
$$

This distribution is centered on the forward beam and has variance $\sigma^{2}$. Ideally, in the case $\sigma \rightarrow 0$, we have $\wp(p) \rightarrow \delta\left(p-p_{f}\right)$ and $\hat{\rho}_{0} \rightarrow\left|p_{f}\right\rangle\left\langle p_{f}\right|$. In order to resolve the forward and Bragg momentum peaks $\sigma$ must be less than one-half. In this paper we set $\sigma=0.1$. If the source of atoms is a beam then this can be obtained by collimating slits [18]. If the atoms are prepared in a trap then this level of cooling can be achieved using velocity selective coherent population trapping or Raman cooling [22]. 
We are interested in the effect of phase noise on the Bragg scattering system. The evolution of the ensemble of atoms is given by Eq. (2.2). Provided both the coupling $\kappa$ and the phase noise diffusion rate $D$ are small, states other than $|p\rangle$ and $|p+1\rangle$ are not significantly populated and scattering by the light field can be analytically treated using a two-state approximation. The master equation (2.2) describing the evolution between the states $|p\rangle$ and $|p+1\rangle$ then reduces to

$$
\frac{d \bar{\rho}}{d t}=i \Delta(p)\left[\hat{\sigma}_{z}, \bar{\rho}\right]-i \kappa\left[\hat{\sigma}_{x}, \bar{\rho}\right]-\frac{D}{2}\left[\hat{\sigma}_{z},\left[\hat{\sigma}_{z}, \bar{\rho}\right]\right],
$$

where $\bar{\rho}$ is a $2 \times 2$ matrix, and $\Delta(p)=p+1 / 2$ is the momentum-dependent detuning from the exact Bragg condition $p=p_{f}$. Here $\hat{\sigma}_{z}$ and $\hat{\sigma}_{x}$ are the usual Pauli spin matrices with respect to the basis $|\uparrow\rangle=|p\rangle$ and $|\downarrow\rangle=|p+1\rangle$. Here and in what follows we use the bar notation to denote the twolevel approximation. This system realizes the completely solvable TLM which has been used previously to study the quantum Zeno effect [6]. It has also appeared in the context of collisional damping of a two-level atom in quantum optics [23] and in studies of nuclear magnetic resonance [24].

The scattering probabilities are most conveniently given in terms of the atomic inversion $Z(p, t)=[\bar{\rho}(p, p)$ $-\bar{\rho}(p+1, p+1)] / 2$ with solution given by Torrey [24]. The total population that has scattered into the Bragg beam is obtained by integrating over the initial state mixture, giving

$$
\bar{P}_{b}(t)=\frac{1}{2}-\int_{-\infty}^{\infty} d p Z(p, t) \wp(p) .
$$

Strictly speaking the above integration should be over the range of momenta in the forward beam, $p \in[-1,0]$. To obtain analytic solutions we note that the initial distribution $\wp(p)$ is negligible outside this range and we extend the limits without loss of accuracy. These analytic solutions demonstrate the Zeno effect which we discuss in the next section.

\section{THE QUANTUM ZENO EFFECT}

The quantum Zeno effect is the inhibition of the free evolution of a system when that system is subject to decohering measurements. We compare the two-state model with a numerical simulation of atomic motion in the stochastic potential. We model a gas of atoms by choosing ten minimum uncertainty states with mean positions distributed uniformly across a single period of the optical potential. The variance in momentum of each state is $\sigma^{2}$ with mean centered on $p_{f}$. Each of these states is then evolved according to the stochastic Hamiltonian Eq. (2.1) to give a history $\left|\psi_{c}(t)\right\rangle$. We use the first-order split-operator method to integrate the Schrödinger equation forward by a time $d t$. A phase trajectory is pre-specified by generating random increments $d \phi=\sqrt{D d t} x(t)$. Here $x(t)$ is a Gaussian random variable with zero mean and unit variance [19]. Once the state $\left|\psi_{c}(t)\right\rangle$ is known the population in the Bragg beam is given by the expression

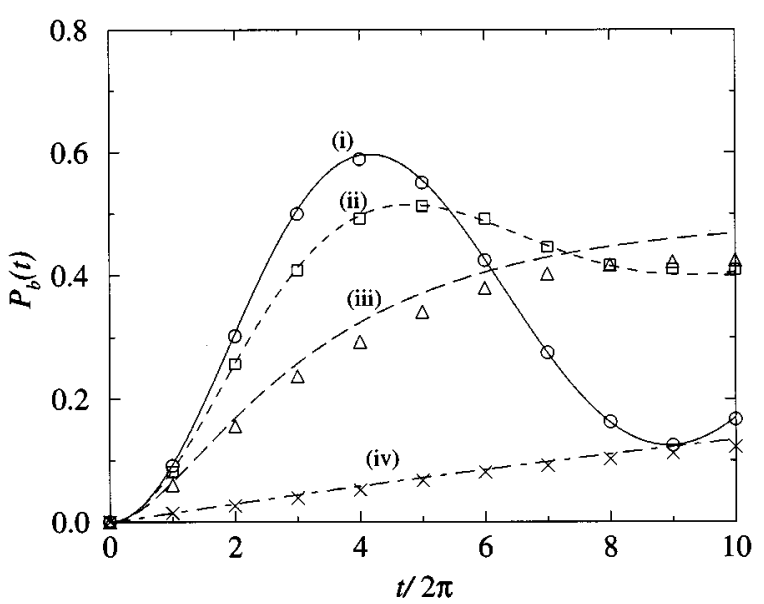

FIG. 1. Fraction of atoms in the Bragg beam as a function of time. The symbols correspond to a numerical solution of the stochastic Hamiltonian equation over 100 noise trajectories and the lines correspond to the two-level approximation. (i) No phase noise $D=0$; (ii) underdamped scattering $D=\kappa$; (iii) critically damped scattering $D=4 \kappa$; (iv) overdamped scattering $D=40 \kappa$.

$$
P_{b}(t)=E\left[\int_{0}^{1} d p\left\langle p\left|\psi_{c}(t)\right| p\right\rangle\right],
$$

where $E[]$ denotes the double average taken over the ensemble of initial states and a large number of phase trajectories. In this paper we found that 100 or 200 noise trajectories were sufficient to allow comparison with the two-state model. The fit between the numerical solution and the twolevel approximation is shown in Fig. 1. Here, we demonstrate that Bragg scattering with phase noise realizes the ideal quantum TLM (3.3) and (4.1).

We use this model to investigate the quantum Zeno effect in which the evolution of the system can be frozen in the initial state. This freezing occurs in the limit $D \rightarrow \infty$. In approaching this limit, the system passes through several dynamical regimes. Although in our solution of the TLM we have integrated over the initial distribution of momenta, the behavior of the Torrey solutions when $p=p_{f}[\Delta(p)=0]$ is sufficient to illustrate these regimes, and the quantum Zeno effect. The finite spread of the initial momentum does not qualitatively change this discussion. When $D=0$ Pendellösung oscillations are apparent in the Bragg beam population (solid line, Fig. 1). The finite width of the initial momentum distribution prevents the population from completely returning to the forward beam. As noise increases to lie between $0<D<4 \kappa$, the population oscillations are underdamped (dashed line, Fig. 1). Critical damping appears when $D=4 \kappa$ as shown in the long-dashed line of Fig. 1. For large noise, the system is overdamped. The signature of a perfect Zeno effect is the absence of population in the Bragg beam. This appears only in the $D \rightarrow \infty$ limit. For nonzero and finite $D$, the system evolves to a mixed state with half the population in each beam. The quantum Zeno effect occurs on time scales small compared to the equilibrium time, and appears when the effect of the phase noise is sufficient to inhibit the growth of population in the Bragg beam. This is already apparent from Fig. 1. Nonetheless the two-state approximation makes detailed predictions about the short-time evolu- 


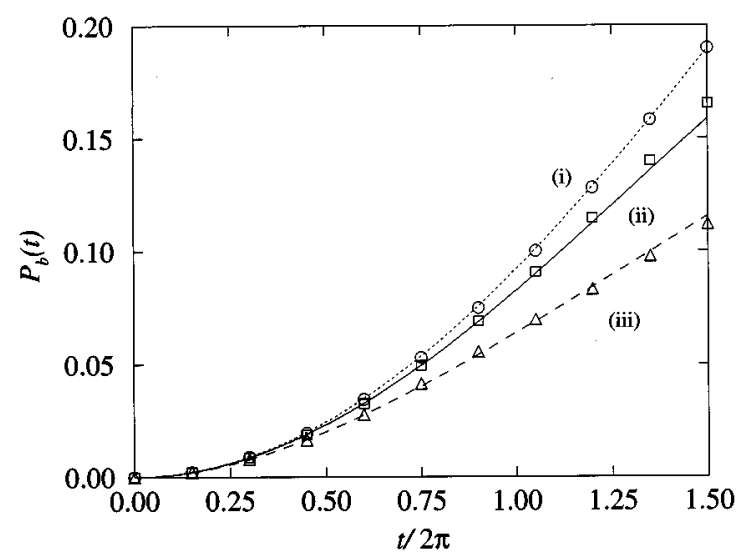

FIG. 2. Fraction of atoms in the Bragg beam as a function of time for underdamped scattering. The symbols correspond to numerical simulations over 100 noise trajectories and the lines are the Zeno effect for a two-state system. (i) $D=0$; (ii) $D=\kappa$; (iii) $D=4 \kappa$.

tion of the Bragg beam and these predictions should be testable by experiments. For underdamped scattering the twostate approximation predicts

$$
\bar{P}_{b}(t) \approx \frac{\kappa^{2}}{4} t^{2}-\frac{D \kappa^{2}}{24} t^{3}-\frac{\kappa^{2}\left(4 \sigma^{2}+4 \kappa^{2}-D^{2}\right)}{192} t^{4} .
$$

The short-time population evolution should be experimentally accessible, and we show this regime in Fig. 2. The two-level model accurately predicts population evolution for moderate values of the noise.

We see that as the phase diffusion rate increases the term cubic in time will suppress the growth of population in the Bragg beam $[4,5]$. Indeed, in the limit $t \rightarrow 0$, we see that population suppression can only occur as $D \rightarrow \infty$. In this case, the time scale for quadratic evolution becomes progressively smaller, and for longer times, populations evolve linearly. For overdamped scattering, the Bragg beam population grows linearly in time. The rate predicted by the TLM $\gamma$ is given by

$$
\gamma=\frac{\kappa^{2}}{D}\left(1-\frac{4 \sigma^{2}}{D^{2}}\right)
$$

showing that the rate of population increase in the Bragg beam is, to first order, inversely proportional to the phase diffusion rate. In Fig. 3 we compare the prediction of Eq. (4.3) with a linear regression analysis of results obtained from our numerical simulations. We find good agreement with the TLM.

\section{COHERENT SUPPRESSION OF BRAGG SCATTERING}

Suppression of Bragg scattering due to the motion of the standing wave does not in itself imply the Zeno effect. It is well known that any deviation in the velocity of the standing wave, whether random or deterministic, causes an effective mismatch of the Bragg condition and can lead to a drastic reduction in the scattering probability. It is therefore important to establish what constitutes an unambiguous signature of the Zeno effect. In this section we treat three cases where

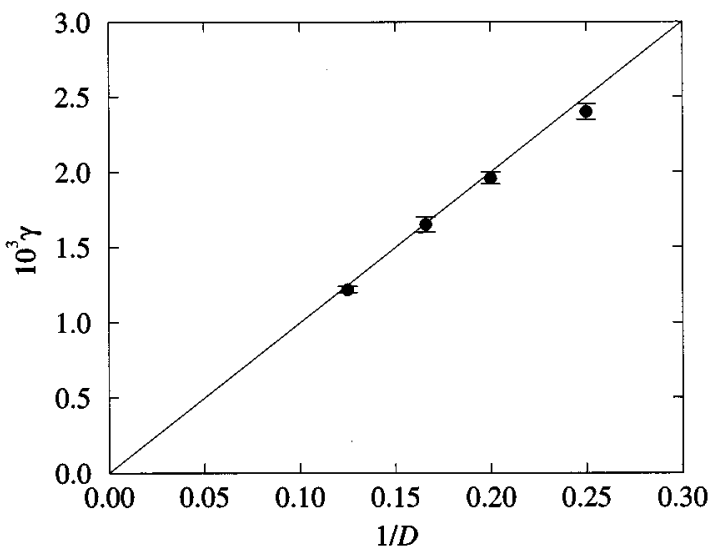

FIG. 3. Rate of increase of atoms in the Bragg beam $\gamma$ as a function of $1 / D$ for overdamped scattering. The symbols correspond to numerical simulations using 100 noise trajectories and the lines are the Zeno effect for a two-state system.

the phase is deterministic and therefore does not lead to decoherence in the ensemble picture. Although Bragg scattering is suppressed in all three cases we show that an examination of the short-time dynamics never reproduces the linear behavior characteristic of the TLM for large $D$.

Restricting our analysis to the two states $p= \pm 1 / 2$ we can derive the following closed expression for the rate of change of the Bragg population when $\phi(t)$ is deterministic.

$$
\dot{P}_{b}(t)=-\frac{\kappa^{2}}{2} \int_{0}^{t} d t^{\prime} \cos \left[\phi(t)-\phi\left(t^{\prime}\right)\right]\left[2 P_{b}\left(t^{\prime}\right)-1\right] .
$$

In deriving this equation we have assumed that $P_{b}(0)=0$. Now consider times short enough that the population in Bragg beam is small compared to $1 / 2$. To a first order we can therefore neglect $P_{b}(t)$ on the right-hand side.

$$
\text { A. } \phi(t)=v t
$$

This case describes a standing wave moving with constant velocity. Substituting for $\phi(t)$ in Eq. (5.1) the integral can be evaluated to give

$$
\dot{P}_{b}(t) \approx \frac{\kappa^{2}}{2 v} \sin (v t)
$$

Therefore for $v>\kappa^{2}$ the Bragg scattering will be suppressed. Notice though that the rate $\dot{P}_{b}$ is sinusoidal in time with a frequency that increases with $v$.

$$
\text { B. } \phi(t)=(D t)^{1 / 2}
$$

This case is closely related to a standing wave with a random phase given by a Wiener process. It corresponds to replacing the random phase by the rms deviation from the initial value. Equation (5.1) then gives

$$
\dot{P}_{b}(t) \approx \frac{2 \kappa^{2}}{D} \sin ^{2} \sqrt{D t} .
$$


For large $D>\kappa^{2}$ Bragg scattering will be suppressed but once again the presence of coherent evolution leads to a sinusoidal variation of $\dot{P}_{b}$ with time at a frequency that increases with $D$.

$$
\text { C. } \phi(t)=\lambda \sin (\omega t)
$$

In our last example we model the suppression of Bragg scattering in a phase modulated standing wave as reported in Ref. [18]. For $\lambda \gg 1$ asymptotic expression can be derived for the two cases $\omega t<\pi / 2$ and $\omega t \gg \pi / 2$. We find

$$
\dot{P}_{b}(t) \approx\left\{\begin{array}{l}
\frac{\kappa^{2}}{2 \omega \lambda} \sin (\lambda \sin \omega t), \quad \omega t<\pi / 2 \\
\frac{\kappa^{2} t \cos (\lambda-\pi / 4)}{\sqrt{2 \pi \lambda}} \cos (\lambda \sin \omega t), \quad \omega t \gg \pi / 2 .
\end{array}\right.
$$

For large $\lambda$ Bragg scattering will be suppressed. The dynamics of $\dot{P}_{b}$ now displays a complicated nonlinear dependence on time.

\section{CONCLUSION}

We have proposed an experiment to observe the quantum Zeno effect in atomic Bragg scattering. Our proposal is based upon the use of classical noise to duplicate the effect of a continuous measurement of atomic momentum on the momentum statistics. Although along each noise trajectory the dynamics is Hamiltonian when averaged over an en- semble of noise trajectories the density operator shows decoherence in the momentum representation. We have shown that for realistic experimental parameters an exactly solvable model in measurement theory can be realized in the context of atom optics. We find good agreement with this model and a full numerical simulation of atomic scattering in the presence of phase noise. We have also compared our results to cases where Bragg scattering is coherently suppressed. None of these cases can reproduce the linear increase in the Bragg beam population predicted by the Zeno effect.

The original idea of the Zeno effect by Misra and Sudarshan [4] was to prevent the decay of an unstable quantum system by performing repeated measurements during the short-time nonexponential stage of the evolution. No such effect has been observed as yet. It is therefore natural to ask whether atom optics might also be the ideal testing ground for this more elusive form of the Zeno effect. Laser cooled atoms in an accelerated standing wave would be the obvious system to examine as atoms in the lowest energy band are known to be unstable and exhibit Landau-Zener tunneling [3]. Our preliminary theoretical treatments of this system subject to various types of noise suggest that suppression of decay for short times should be possible, provided the type of noise is chosen correctly. More extensive numerical modeling is being pursued.

\section{ACKNOWLEDGMENT}

One of us (S.D.) would like to acknowledge valuable conversations with M. G. Raizen.
[1] M. H. Anderson, J. R. Ensher, M. R. Matthews, C. E. Wieman, and E. A. Cornell, Science 269, 198 (1995); K. B. Davis, M.-O. Mewes, M. R. Andrews, N. J. van Druten, D. S. Durfee, D. M. Kurn, and W. Ketterle, Phys. Rev. Lett. 75, 3969 (1995).

[2] F. L. Moore, J. C. Robinson, C. F. Bharucha, Bala Sundaram, and M. G. Raizen, Phys. Rev. Lett. 75, 4598 (1995).

[3] C. F. Bharucha, K. W. Madison, P. R. Morrow, S. R. Wilkinson, Bala Sundaram, and M. G. Raizen, Phys. Rev. A 55, 857 (1997).

[4] B. Misra, E. C. G. Sudarshan, J. Math. Phys. (N.Y.) 18, 756 (1977).

[5] A. Peres, Am. J. Phys. 48, 931 (1980).

[6] G. J. Milburn, J. Opt. Soc. Am. B 5, 1317 (1988).

[7] W. M. Itano, D. J. Heinzen, J. J. Bollinger, and D. J. Wineland, Phys. Rev. A 41, 2295 (1990).

[8] M. J. Gagen and G. J. Milburn, Phys. Rev. A 45, 5228 (1992).

[9] G. J. Milburn and M. J. Gagen, Phys. Rev. A 46, 1578 (1992).

[10] E. Block and P. R. Berman, Phys. Rev. A 44, 1466 (1991).

[11] V. Frerichs and A. Schenzle, Phys. Rev. A 44, 1962 (1991).

[12] M. J. Gagen and G. J. Milburn, Phys. Rev. A 47, 1467 (1993).

[13] W. H. Zurek, Phys. Rev. D 24, 1516 (1981).
[14] S. Dyrting and G. J. Milburn, Quantum Semiclass. Opt. 8, 541 (1996).

[15] S. Dürr, S. Kunze, and G. Rempe, Quantum Semiclass. Opt. 8, 531 (1996).

[16] A. P. Kazentsev, G. I. Surdutovich, and V. P. Yakovlev, Mechanical Action of Light on Atoms (World Scientific, Singapore, 1990).

[17] F. L. Moore, J. C. Robinson, C. Bharucha, P. E. Williams, and M. G. Raizen, Phys. Rev. Lett. 73, 2974 (1994).

[18] Stefan Bernet, Markus Oberthaler, Roland Abfalter, Jörg Schmiedmayer, and Anton Zeilinger, Quantum Semiclass. Opt. 8, 497 (1996).

[19] C. W. Gardiner, Handbook of Stochastic Methods (Springer, New York, 1985).

[20] A. J. Lichtenberg and M. A. Lieberman, Regular and Chaotic Dynamics (Springer, New York, 1992).

[21] P. J. Martin, B. G. Oldaker, A. H. Miklich, and D. E. Pritchard, Phys. Rev. Lett. 60, 515 (1988).

[22] Craig Savage, Aust. J. Phys. 49, 745 (1996).

[23] L. Allen and J. H. Eberly, Optical Resonances and Two-Level Atoms (Wiley, New York, 1975).

[24] H. C. Torrey, Phys. Rev. 76, 1059 (1949). 